Marc Tardieu, MD, PhD

Brenda Banwell, MD

Jerry S. Wolinsky, MD

Daniela Pohl, MD, PhD

Lauren B. Krupp, MD

Correspondence to

Dr. Tardieu:

marc.tardieu@bct.aphp.fr

\title{
Consensus definitions for pediatric MS and other demyelinating disorders in childhood
}

\section{ABSTRACT}

In light of the published 2012 International Pediatric Multiple Sclerosis Group definitions for pediatric multiple sclerosis (MS) and related disorders and given that pediatric-onset MS is now formally included in the 2010 McDonald criteria for MS, we sought to review these criteria and summarize their application in children with acquired CNS demyelination. In addition, proposals are made for definitions of no evidence of disease activity and inadequate treatment response that are important because of new therapeutic options and trials. Neurology ${ }^{\circledR} \mathbf{2 0 1 6 ; 8 7}$ (Suppl 2):S8-S11

\section{GLOSSARY}

ADEM = acute disseminated encephalomyelitis; ARR = annualized relapse rate; CIS = clinically isolated syndrome; DIS = dissemination of inflammatory lesions in space; DIT = dissemination of inflammatory lesions in time; EDSS = Expanded Disability Status Scale; IPMSSG = International Pediatric Multiple Sclerosis Study Group; MOG = myelin oligodendroglial glycoprotein; $\mathbf{M S}=$ multiple sclerosis; NEDA = no evidence of disease activity; NMO = neuromyelitis optica; RIS = radiologically isolated syndrome.

The International Pediatric Multiple Sclerosis Study Group (IPMSSG) proposed definitions for pediatric multiple sclerosis (MS), acute disseminated encephalomyelitis (ADEM), neuromyelitis optica (NMO), and clinically isolated syndromes (CIS) to facilitate research providing consistency in terminology. ${ }^{1}$ In 2012, these definitions were revised to incorporate advances in research and to include components of the 2010 revision of the McDonald criteria. ${ }^{1,2}$ The cornerstone of a diagnosis of MS, both in adults and children, is the demonstration of dissemination of inflammatory lesions in space (DIS) and in time (DIT) in association with clinical symptoms consistent with acquired CNS demyelination and after the careful exclusion of numerous differential diagnoses. Incorporation of the $2010 \mathrm{McDonald}$ criteria (that in addition to confirming relapsing-remitting MS diagnosis on the basis of new clinical or MRI disease activity over time, also permit MS diagnosis at the time of a first clinical MS attack if the MRI fulfils specific DIS and DIT criteria) to the revised IPMSSG definitions facilitated earlier diagnosis of pediatric MS and provided inclusion criteria for new therapeutic trials (table).

Our aim is to review application of the 2012 IPMSSG definitions in pediatric cohorts with acute demyelinating syndromes, discuss clinical scenarios that do not conform to the current definitions, suggest concepts regarding definition of adequate and inadequate treatment responses, and consider future research directions.

EVALUATIONS OF 2012 IPMSSG AND 2010 MCDONALD CRITERIA TO DIAGNOSE MS IN CHILDREN Clinically isolated syndromes. Five reports evaluated the accuracy of the 2010 McDonald criteria to diagnose MS in children, with similar results despite methodologic differences. ${ }^{3-7}$ At time of first CIS, after application of the $2010 \mathrm{McD}$ onald criteria to the initial brain MRI, the diagnosis of MS was met in 53\%-63\% of the children who subsequently developed a clinical relapse. DIT was less frequently present at initial brain MRI than DIS, which was observed in more than $80 \%$ of children. Interestingly, the inclusion of spinal cord imaging did not increase accuracy of MS diagnosis. ${ }^{4,6} \mathrm{~A}$ follow-up MRI performed at 3 months, demonstrating new lesions, increased the identification of MS to $84 \%-100 \%$ of children who later had clinical relapse. ${ }^{4,6}$

From the National Reference Center for Inflammatory Diseases of the Brain (M.T.), Hôpitaux Universitaires Paris-Sud, University Paris-Sud, France; Perelman School of Medicine (B.B.), The Children's Hospital of Philadelphia, University of Pennsylvania; University of Texas Health Science Center at Houston (J.W.); Department of Neurology (D.P.), Children's Hospital of Eastern Ontario, University of Ottawa, Canada; and Lourie Center for Pediatric MS (L.B.K.), Stony Brook Children's, NY.

Go to Neurology.org for full disclosures. Funding information and disclosures deemed relevant by the authors, if any, are provided at the end of the article. 
Table Summary of 2012 International Pediatric Multiple Sclerosis Study Group definitions for pediatric multiple sclerosis (MS) and immune-mediated CNS demyelinating disorders ${ }^{1}$

Pediatric clinically isolated syndrome (CIS) (all are required)

A clinical CNS event with presumed inflammatory demyelinating cause.

Absence of a clinical history of CNS demyelinating disease (if any, see pediatric MS).

No encephalopathy except as readily explained by fever.

Criteria for MS diagnosis on baseline MRI are not met.

Pediatric acute disseminated encephalomyelitis (ADEM) (all are required)

A first polyfocal, clinical CNS event with presumed inflammatory demyelinating cause.

An encephalopathy that cannot be explained by fever.

No new clinical or MRI findings 3 months or more after onset.

Brain MRI is abnormal during the acute ( 3 months) phase with typically diffuse, poorly demarcated large lesions involving predominantly the cerebral white matter.

Pediatric MS (any of the following)

Two or more CIS separated by more than 30 days involving more than one area of CNS.

One CIS associated with MRI findings consistent with criteria of dissemination in space (DIS) and in which a follow-up MRI shows at least one new lesion consistent with dissemination in time (DIT) criteria.

One ADEM attack followed by $1 \mathrm{CIS} 3$ or more months after symptom onset that is associated with new MRI findings consistent with criteria for DIS.

A CIS whose MRI findings are consistent with criteria for DIS and DIT (at least 1 T2 lesion in at least 2 of 4 areas: spinal cord, infratentorial, juxtacortical, and periventricular [DIS] associated with a simultaneous presence of asymptomatic gadolinium-enhancing and nonenhancing lesions [DIT] if the patient is $\geq 12$ years old) (revision proposed).

Pediatric neuromyelitis optica (revised: See "Neuromyelitis optica sectrum disorders in children and adolescent" on page S59.) at 3 months ${ }^{3,6}$ suggest that this time interval is both efficient (most patients will be recognized) and safe (treatment will not be excessively delayed). As such, we recommend a repeat MRI 3 months after a diagnosis of pediatric CIS.

3. Other diagnosis such as vasculitis might fulfil DIS and DIT criteria: a clinically relevant history is mandatory to diagnose MS.

Acute disseminated encephalomyelitis. In children presenting with ADEM (in whom the presence of multiple lesions is likely to meet DIS and the presence of enhancing and nonenhancing lesions would also meet DIT), the criteria for a MS diagnosis using baseline DIT and DIS imaging criteria are not appropriate. ${ }^{5}$ As an ADEM-like attack is the first MS event in only $5 \%-15 \%$ of children, ${ }^{1}$ the diagnosis of MS in such children requires a second non-ADEM attack as well as either further MRI findings of clinically silent new lesions or a third attack also not meeting criteria for ADEM.

Radiologically isolated syndrome (RIS). RIS refers to individuals with incidental MS-typical MRI findings in whom clinical history or signs of MS are lacking. The following adult criteria ${ }^{8}$ are believed to be appropriate for pediatric RIS but should be validated:

1. MRI showing ovoid, well-circumscribed, and homogenous T2 hyperintensities fulfilling at least 3 Barkhof criteria (at least 1 gadoliniumenhancing lesion or 9 T2-hyperintense lesions; at least 1 infratentorial lesion; at least 1 juxtacortical lesion; at least 3 periventricular lesions).

2. No historical account of remitting symptoms of neurologic dysfunction indicating MS.

3. MRI findings do not account for symptoms for which the individual was imaged.

4. MRI findings are not better explained by another disease process.

In adults, male sex and an MRI lesion in the spinal cord increase the likelihood that RIS will be associated with a subsequent clinical attack ${ }^{9}$ but data are lacking for children. Published experience of pediatric RIS is limited to 5 cases (2-13 years): none developed a clinical event during 5-15 years of follow-up. ${ }^{10}$ The prevalence of cognitive deficits in children with RIS is unknown but worthy of investigation as adults with RIS have a cognitive profile similar to those with remitting-relapsing MS. ${ }^{11}$

CNS-directed antibodies as potential biomarkers for distinct demyelinating disease phenotypes. The identification of anti-aquaporin 4 antibodies as a relevant biomarker for neuromyelitis optica spectrum disorders emphasizes the potential for antibodies to aid in the diagnosis of CNS inflammatory disorders. Key considerations for antibodies as biomarkers include 
(1) fidelity with clinical features (see chapter 9); (2) persistent detection if such persistence distinguishes a specific disorder from transient immune recognition of multiple CNS antigens; and (3) pathogenicity of the antibodies within the CNS (required if the antibodies are thought to contribute to the disease directly). The assays used to measure antibodies must also be carefully validated (the increased sensitivity of cellbased assays now used for detection of aquaporin-4 antibodies emphasizes this point).

Anti-myelin oligodendroglial glycoprotein (MOG) antibodies have been detected in 18\%-35\% of children with a first acute episode of inflammatory demyelination" ${ }^{12-14}$ (see "Immunopathophysiology of pediatric CNS inflammatory demyelinating disease" on page S12). The transient detection of anti-MOG antibodies is more frequent in children at onset of ADEM, optic neuritis, and relapsing optic neuritis, and anti-MOG positivity predicts a non-MS disease course. ${ }^{13-16}$ MOG-positive children have clinical and radiologic features typical for ADEM and elevated lymphocyte counts in CSF, suggesting a high degree of inflammation. ${ }^{16,17}$ Persistence of anti-MOG antibodies has been associated with MS, although fewer than 25\% of pediatric patients with MS have detectable antiMOG antibodies. ${ }^{12}$ Demonstrating the pathogenicity of anti-MOG antibodies and following larger cohorts of pediatric patients who have been tested for them are needed for the presence of MOG antibodies to serve as a disease-specific biomarker and to guide patient care.

DEFINITION OF NO EVIDENCE OF DISEASE ACTIVITY (NEDA) IN PEDIATRIC MS With multiple effective MS therapies, NEDA has become an MS treatment goal. ${ }^{18,19}$ Whether the definition of inactive disease should focus solely on inflammatory disease activity or also include brain volume changes, cognition, or other measures remains unresolved. ${ }^{20-22}$ Defining the more global aspects of MS effect, such as the effect on brain growth, is even more challenging for pediatric than adult MS, since estimations of pathologic brain volume changes during development will require complex algorithms that incorporate expected physiologic brain growth. ${ }^{23,24}$ Incorporating the effects of MS on cognitive development is also difficult. $^{25}$ Although specific pediatric NEDA criteria are still under development, the authors agree that the following elements are critical-absence of new, enlarging, or enhancing lesions on MRI; clinical relapses; and confirmed disability progression — and suggest that consideration be given to preservation of ageexpected global and regional brain growth and to age-expected cognitive maturation and function.

DEFINITION OF TREATMENT RESPONSE Clinical relapse remains the gold standard metric for relapsing-remitting MS disease activity, and is a required primary endpoint for Food and Drug Administration-supported clinical trials in MS. Treatment-naive pediatric patients with MS have an annualized relapse rate of $0.9-3.2$ in the first 2 years following the first attack and of $0.5-0.8$ in the next 3-5 years. ${ }^{26,27}$ Assuming that first-line disease-modifying therapies in children should provide a reduction in relapses similar to the $30 \%-40 \%$ observed in adults, a treatment goal would be to initially achieve an annualized relapse rate (ARR) lower than 0.6 relapses per year (or roughly 1 relapse over the first 2 years of disease) and $\leq 0.35$ between years $2-5$ post disease onset. ${ }^{26,27}$ Treatment benefit in adult MS trials often includes absence of sustained Expanded Disability Status Scale (EDSS) increase. This metric is less appropriate in pediatric-onset MS as EDSS rarely worsens during the first 10 years following disease onset. ${ }^{28}$ Reduction in new T2-bright or gadoliniumenhancing T1-dark lesions on MRI is another possible measure of treatment benefit that can be used in children since a serial MRI study of pediatric patients with MS showed a mean of 9 new lesions 6 months following the first attack. ${ }^{29}$

A proposed definition for an inadequate treatment response (in a fully compliant patient) is an increase or lack of reduction in ARR, 2 or more gadoliniumenhancing lesions on MRI or accrual of more than 2 new lesions when compared to the pretreatment period, and 2 or more confirmed relapses within a 12 -month period or less. ${ }^{30}$

Cognitive impairment, fatigue, and emotional health are important MS symptoms that should be considered when deciding to start or change MS treatment.

PERSPECTIVES We propose to incorporate in IPMSSG criteria the 2010 McDonald criteria for MS for children of all ages when MRI-defined DIT and DIS requirements for MS are present at the first attack provided the sentinel event is consistent with acute demyelination and the attack does not conform to the criteria of ADEM. All other definitions are unchanged, except for the NMO spectrum disorders (See "Neuromyelitis optica sectrum disorders in children and adolescent" on page S59). Future goals are to better define reliable prognostic markers to define subgroups of patients and better understand and measure the degenerative component of MS in children, particularly in reference to cognitive functioning, age-expected brain growth, and diffuse neural networks.

\section{AUTHOR CONTRIBUTIONS}

All authors contributed to the elaboration of the content and revised the manuscript equally. 


\section{STUDY FUNDING}

This supplement is made possible by funding from the MS Cure Fund, Danish MS Society, German MS Society, Italian MS Association, MS International Federation, MS Research Foundation (Netherlands), National MS Society (USA) and Swiss MS Society.

\section{DISCLOSURE}

M. Tardieu received honoraria or consultation fees from Novartis, Sanofi, Teva, and Biogen. B. Banwell serves as a central MRI reviewer for a clinical trial for Novartis. J. Wolinsky and D. Pohl report no disclosures relevant to the manuscript. L. Krupp received honoraria or consultation fees from Biogen, Novartis, Teva Neurosciences, Pfizer, Projects in Knowledge, Providence Health Services, and Multicell; royalty payments from Avanir Pharmaceuticals, Abbvie Inc., Osmotica Pharmaceutical Corp., and Demos Medical Publishing; and received research funding from Novartis, Teva Neurosciences, Biogen Idec, Genentech, National Multiple Sclerosis Society, Department of Defense, National Institutes of Health, and the Lourie Foundation. Go to Neurology.org for full disclosures.

Received August 19, 2015. Accepted in final form March 1, 2016.

\section{REFERENCES}

1. Krupp LB, Tardieu M, Amato MP, et al. International Pediatric Multiple Sclerosis Study Group criteria for pediatric multiple sclerosis and immune-mediated central nervous system demyelinating disorders: revisions to the 2007 definitions. Mult Scler 2013;19:1261-1267.

2. Poman $\mathrm{CH}$, Reingold SC, Banwell B, et al. Diagnostic criteria for multiple sclerosis: 2010 revisions to the McDonald criteria. Ann Neurol 2011;69:292-302.

3. Sedani S, Lim MJ, Hemingway C, Wassmer E, Absoud M. Paediatric multiple sclerosis: examining utility of the McDonald 2010 criteria. Mult Scler 2012;18:679-682.

4. Kornek B, Schmitl B, Vass K, et al. Evaluation of the 2010 McDonald multiple sclerosis criteria in children with a clinically isolated syndrome. Mult Scler 2012;18:1768-1774.

5. Sadaka Y, Verhey LH, Shroff MM, et al. 2010 McDonald criteria for diagnosing pediatric multiple sclerosis. Ann Neurol 2012;72:211-223.

6. Hummel H-M, Brück W, Dreha-Kulaczewski S, Gärtner J, Wuerfel J. Pediatric onset multiple sclerosis: McDonald criteria 2010 and the contribution of spinal cord MRI. Mult Scler 2013;19:1330-1335.

7. Williams MT, Tapos DO, Juhasz C. Use of the 2010 McDonald criteria can facilitate early diagnosis of pediatric multiple sclerosis in a predominantly Black cohort. Pediatr Neurol 2014;51:826-830.

8. Okuda DT, Mowry EM, Beheshtian A, et al. Incidental MRI anomalies suggestive of multiple sclerosis: radiologically isolated syndrome. Neurology 2009;72:800-805.

9. Okuda DT, Siva A, Kantarci O, et al. Radiologically isolated syndrome: 5-year risk for an initial clinical event. PloS One 2014;9:e90509.

10. Eraksoy M, Yapici Z, Tekturk P, Kurtuncu M, Ozcan H. Radiologically isolated syndrome in children: report of 5 children with long-term follow-up. Neurology 2014;82 (suppl):P2.243.

11. Lebrun C, Blanc F, Brassat D, et al, On behalf of CFSEP cognitive function in radiologically isolated syndrome. Mult Scler 2010;16:919-925.

12. Pröbstel AK, Dormair K, Bittner R, et al. Antibodies to MOG are transient in childhood ADEM. Neurology 2011;77:580-588.
13. Hacohen Y, Absoud M, Deiva K, et al. Myelin oligodendrocytes glycoprotein antibodies predict a non-MS demyelination course in children. Neurol Neuroimmunol Neuroinflamm 2015;2:e81.

14. Ketelslegers IA, VanPelt DE, Bryde S, et al. Anti-MOG antibodies plead against MS diagnosis in an acquired demyelinating syndromes cohort. Mult Scler 2015;12: 1513-1520.

15. Reindl M, Rostasy K. MOG antibody-associated diseases. Neurol Neuroimmunol Neuroinflamm 2015;2:e60.

16. Fernandez-Carbonell C, Vargas-Lowy D, Masallam A, et al. Clinical and MRI phenotype of children with MOG. Mult Scler 2016;22:2174-2184.

17. Baumann M, Sahin K, Lechner C, et al. Clinical and neuroradiological differences of paediatric acute dissemination encephalomyelitis with and without antibodies to the myelin oligodendrocyte glycoprotein. J Neurol Neurosurg Psychiatry 2015;86:265-272.

18. Bevan CJ, Cree BA. Disease activity free status: a new end point for a new era in multiple sclerosis clinical research? JAMA Neurol 2014;71:269-270.

19. Stangel M, Penner IK, Kallmann BA, Lukas C, Kieseier BC. Towards the Implementation of "no evidence of disease activity" in multiple sclerosis treatment: the multiple sclerosis decision model. Ther Adv Neurol Disord 2015;8:3-13.

20. Havrdova E, Galetta S, Stefoski D, Comi G. Freedom from disease activity in multiple sclerosis. Neurology 2010;74(suppl 3):S3-S7.

21. Lublin F. Disease activity free Status in MS. Mult Scler Relat Dis 2012;1:6-7.

22. Rotstein D, Healy BC, Malik MT, Chitnis T, Weiner HL. Evaluation of no evidence of disease activity in a 7-year longitudinal multiple sclerosis cohort. JAMA Neurol 2015;72:152-158.

23. Aubert-Broche B, Fonov V, Narayanan S, et al. Onset of multiple sclerosis before adulthood leads to failure of ageexpected brain growth. Neurology 2014;83:2140-2146.

24. Mesaros S, Rocca MA, Absinta M, et al. Evidence of thalamic gray matter loss in pediatric multiple sclerosis. Neurology 2008;70:1107-1112.

25. Amato MP, Goretti B, Ghezzi A, et al. Neuropsychological features in childhood and juvenile multiple sclerosis: fiveyear follow-up. Neurology 2014;83:1432-1438.

26. Chitnis T, Tardieu M, Amato MP, et al. International Pediatric MS Study Group clinical trials summit. Neurology 2013;80:1161-1168.

27. Banwell B, Bar-Or A, Giovannoni G, et al. Therapies for multiple sclerosis: considerations in the pediatric patient. Nat Rev Neurol 2011;7:109-122.

28. Renoux C, Vukusic S, Mikaeloff Y, et al. Natural history of multiple sclerosis with childhood onset. N Engl J Med 2007;356:2603-2613.

29. Verhey LH, Branson HM, Shroff MM, et al. MRI parameters for prediction of multiple sclerosis diagnosis in children with acute CNS demyelination: a prospective national cohort study. Lancet Neurol 2011;10: 1065-1073.

30. Chitnis T, Tenembaum S, Banwell B, et al. Consensus statement: evaluation of new and existing therapeutics for pediatric multiple sclerosis. Mult Scler 2012;18: 116-127. 


\section{Neurology}

\section{Consensus definitions for pediatric MS and other demyelinating disorders in childhood Marc Tardieu, Brenda Banwell, Jerry S. Wolinsky, et al. \\ Neurology 2016;87;S8-S11 \\ DOI 10.1212/WNL.0000000000002877}

This information is current as of August 29, 2016

\section{Updated Information \& Services}

References

Citations

Subspecialty Collections

Permissions \& Licensing

Reprints including high resolution figures, can be found at: http://n.neurology.org/content/87/9_Supplement_2/S8.full

This article cites 30 articles, 1 of which you can access for free at: http://n.neurology.org/content/87/9_Supplement_2/S8.full\#ref-list-1

This article has been cited by 1 HighWire-hosted articles: http://n.neurology.org/content/87/9_Supplement_2/S8.full\#\#otherarticl es

This article, along with others on similar topics, appears in the following collection(s):

Acute disseminated encephalomyelitis

http://n.neurology.org/cgi/collection/acute_disseminated_encephalomy elitis

Multiple sclerosis

http://n.neurology.org/cgi/collection/multiple_sclerosis

Information about reproducing this article in parts (figures,tables) or in its entirety can be found online at:

http://www.neurology.org/about/about_the_journal\#permissions

Information about ordering reprints can be found online:

http://n.neurology.org/subscribers/advertise

Neurology ${ }^{\circledR}$ is the official journal of the American Academy of Neurology. Published continuously since 1951, it is now a weekly with 48 issues per year. Copyright @ 2016 American Academy of Neurology. All rights reserved. Print ISSN: 0028-3878. Online ISSN: 1526-632X.

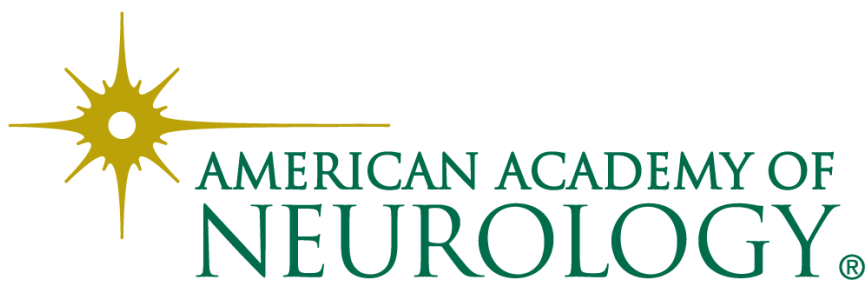

